\title{
Beziehung zwischen Biomasse und Stickstoffgehalt des Phytoplanktons und die daraus ableitbare Anwendung der Bestimmungsmethoden für die Praxis
}

\section{Von Marianne Pavoni}

Eidg. Anstalt für Wasserversorgung, Abwasserreinigung und Gewässerschutz an der ETH, Zürich 


\section{Einleitung}

Die Bestimmung der Biomasse von Planktonorganismen mittels der Zählmethode ist langwierig und bedeutet für den Ausführenden eine Geduldsprobe. Ausserdem können Fehler, die vor allem bei der Berechnung des Planktonvolumens entstehen, die Resultate wesentlich verändern.

Oft wurde daher die Biomasse (bzw. das Zellvolumen oder Zellplasma) mit anderen chemischen oder physikalischen Komponenten der Organismen verglichen oder die Zählmethode durch die Bestimmung eines konstanten Bauelements der organischen Gerüstsubstanz, vor allem des Kohlenstoffs, des Eiweissgehaltes, des organisch gebundenen Stickstoffs oder des Chlorophylls a zu umgehen versucht [12-15, 21]. Diese chemischen Methoden befriedigen jedoch auch nicht immer, da sie für exakte Analysen mit grossem Arbeits- und Apparateaufwand verbunden sind oder in Mikrobereichen zu grosse Fehler aufweisen. Probleme dieser Art bildet zum Beispiel die Bestimmung des Kohlenstoffs [22], der an und für sich eine der besten chemischen Komponenten zur Erfassung der organisch geformten Substanz im See darstellt [15, 21, 22]. Der Kohlenstoffgehalt der Organismen scheint weniger von ihrem physiologischen Zustand und den Umweltsfaktoren als vielmehr von der Art der Organismen abhängigzu sein.

Chlorophyll $a$ hingegen - obgleich eine gebräuchliche Zellkomponente zur Bestimmung des «standing crop» - variiert je nach Entwicklungsstadium und Lebensbedingungen der Algen sehr stark, das heisst, es besteht keine innere Beziehung zwischen der momentanen Chlorophyllmenge und der Biomasse [3].

Ferner wissen wir aus der Literatur, dass auch der Stickstoffgehalt je nach Art und Entwicklungszustand der Organismen variieren kann [7, 15, 22]. EINSELE ([2], S. 440) aber schrieb: "Aus den Ergebnissen der Analysen des Trockensubstanz-, N- und PGehaltes von Planktonproben darf noch ein weiterer für die produktionsbiologische Beurteilung von Seen wichtig erscheinender Schluss gezogen werden, nämlich dass wir im Stickstoffhaushalt von Plankton eine stets brauchbare Proportionszahl seines Eiweissgehaltes haben. Damit besitzen wir auch eine gute Grundlage für die Beurteilung der 〈stehenden〉 Planktonernten, das heisst der zu einem Zeitpunkt vorhandenen Bio- 
massen. " Allerdings erwähnt er auch, dass der Roheiweissgehalt bzw. N-Gehalt von Planktonproben in keinem genau angebbaren konstanten Verhältnis zur Trockensubstanz, auch nicht der aschenfreien, steht.

Nachdem Schmid [18] die Verbesserung der Stickstoffmethode nach Kjeldahl an die Hand genommen und für den Mikrobereich (unter $0,1 \mathrm{mg} / \mathrm{l}$ ) wesentliche Fehlerquellen behoben hat, ergab es sich fast als selbstverständlich, dass wir die Übereinstimmung der berechneten Biomasse mit dem partikulären Stickstoff zu überprüfen begannen. Ziel der vergleichenden Untersuchung war, folgende Fragen abzuklären: a) Welche Beziehung besteht zwischen der berechneten Biomasse und dem partikulären Stickstoffgehalt im See?

b) Lässt sich die Biomassebestimmung durch die Bestimmung des partikulären Stickstoffs ersetzen? Unter welchen Bedingungen?

\section{Methoden}

Analysiert wurden in allen Untersuchungen der partikuläre Stickstoff und die Biomasse. Zusätzlich wurden wenn möglich der partikuläre Phosphorgehalt und als Kontroll- und Bezugsgrösse das Trockengewicht bzw. die organische Trockensubstanz ermittelt. Der Phosphorgehalt wird erst in einer separaten Arbeit eingehender diskutiert werden.

Die Stickstoffanalyse erfolgte nach den Vorschriften von Schmid (1968), im Material aus den Netzzügen durch direkte Kjeldahl-Bestimmung, in den Kulturproben mittels Filtration der Proben durch ein Sartorius-Membranfilter, gewichtskonstant M 50, (Porenweite $0,45 \mu$ ). Der Filterrückstand wurde jeweils mit wenig N-freiem Wasser gewaschen und quantitativ in den Kjeldahlkolben gespült und aufgeschlossen. Der Stickstoffgehalt des Planktons der Schöpfproben (d.h. eigentlich des Sestons) wurde durch die Berechnung der Differenz zwischen dem totalen Kjeldahl-Stickstoff und dem Kjeldahl-Stickstoff im Filtrat ermittelt. Der partikuläre Phosphor wurde nach der Methode von Schmid und AмвüнL [19] erfasst.

Das Trockengewicht wurde erhalten, indem je nach Menge und Herkunft das Material in der Platinschale oder auf einem Sartorius-Membranfilter, gewichtskonstant MF 50, (Porenweite $0,45 \mu$ ), bei $105^{\circ} \mathrm{C}$ im Trockenschrank bis zur Gewichtskon$\operatorname{stanz}$ getrocknet wurde. Anschliessend wurde das getrocknete Material bei höchstens $550^{\circ} \mathrm{C}$ im Muffelofen ebenfalls bis zur Gewichtskonstanz verascht (zur Berechnung der aschenfreien Trockensubstanz).

Zur Berechnung der Biomasse wurden die einzelnen Arten der Netz- und Kulturproben in der Kolkwitz-Kammer und diejenigen der Schöpfproben im Umkehrmikroskop nach UTERMÖHL [23] ausgezählt, ihr Zellvolumen durch Ausmessen der Zelldimensionen und einem der Zelle ähnlichen geometrischen Körper berechnet und mit der Anzahl der gezählten Organismen multipliziert [8].

\section{Material}

Drei Materialtypen wurden untersucht: Netzproben von Phytoplankton aus dem Vierwaldstättersee, Algenmaterial aus Kulturen, ferner Schöpfproben aus dem Vierwaldstättersee. 
Netzproben: Während gut eines Jahres (Mai 1966 bis August 1967) wurde im Vierwaldstättersee monatlich einmal ein Netzzug (Maschenweite $50 \mu$ ) durchgeführt. Zur Sommerstagnationszeit wurde das Netz horizontal in der Tiefe der stärksten Entwicklung von Oscillatoria rubescens $(7,5-12,5 \mathrm{~m})$, in den übrigen Jahreszeiten diagonal ungefähr zwischen 20 und $0 \mathrm{~m}$ gezogen. Auf diese Weise erhielten wir schnell die wichtigsten Netzmassenformen in relativ reiner Form und frei von den vielen Trübungsstoffen, die in den Schöpfproben dagegen oft in grosser Menge vorhanden sind. Die Probe wurde jeweils im Kühlschrank stehengelassen. Dabei trennten sich die spezifisch leichteren Oscillatoria-Fäden von den spezifisch schwereren übrigen Netzplanktonformen ab, so dass wir zwei Fraktionen, eine mit reinem Material von Oscillatoria rubescens und eine andere mit den restlichen, abgesetzten Planktonformen auf die oben erwähnten Komponenten untersuchen konnten. Die grösseren Zooplankter wurden so gut wie möglich wegpipettiert.

Die letztgenannte Fraktion, das heisst die Probe ohne Oscillatoria rubescens setzte sich zur Hauptsache aus den üblichen Netzkieselalgen Tabellaria fenestrata, Synedra acus, Diatoma elongatum und Asterionella formosa zusammen, die mit Ausnahme von drei Monaten stets mehr als 50\% (ja bis zu knapp 100\%) ausmachten (Tab. 1). Durchschnittlich wurden 10 Arten ausgezählt. Noch vorhandene Zooplankter wie haupt-

Tab. 1. Netzzüge (Vierwaldstättersee, Stelle Kreuztrichter), Fraktion mit dem restlichen Netzplankton: Prozentuale Biomasseanteile der Algen und Tiere.

\begin{tabular}{|c|c|c|c|c|c|c|c|c|}
\hline Organismengruppen & $\begin{array}{l}24.5 . \\
1966\end{array}$ & $\begin{array}{l}23.6 . \\
1966\end{array}$ & $\begin{array}{l}15.8 . \\
1966\end{array}$ & $\begin{array}{l}14.9 . \\
1966\end{array}$ & $\begin{array}{l}11.10 . \\
1966\end{array}$ & $\begin{array}{l}7.11 \\
1966\end{array}$ & $\begin{array}{l}7.12 . \\
1966\end{array}$ & \\
\hline \multicolumn{9}{|l|}{ Algen: } \\
\hline Cyanophyta & - & 0,6 & 7 & 2 & 29 & 16 & 0,7 & \\
\hline Chlorophyta & - & 0,6 & 7 & 6 & 0,6 & 1 & 0,7 & \\
\hline Chrysophyceae & 一 & 3 & - & - & - & - & - & \\
\hline Diatomeac & 99 & 95 & 79 & 67 & 8 & 17 & 61 & \\
\hline Peridineae & - & - & 4 & 20 & 58 & 59 & 37 & \\
\hline \multicolumn{9}{|l|}{$\begin{array}{l}\text { Perıdıneae } \\
\text { Tiere: }\end{array}$} \\
\hline $\begin{array}{l}\text { Rotatoria, Phyllopoda, } \\
\text { Copepoda }\end{array}$ & 0,5 & 0,8 & 3 & 5 & 5 & 6 & 0,7 & \\
\hline Total & 99,5 & 100 & 100 & 100 & 100,6 & 99 & 100,1 & \\
\hline Organismengruppen & $\begin{array}{l}5.1 \\
1967\end{array}$ & $\begin{array}{l}30.1 \\
1967\end{array}$ & $\begin{array}{l}27.2 . \\
1967\end{array}$ & $\begin{array}{l}28.3 . \\
1967\end{array}$ & $\begin{array}{l}24.4 . \\
1967\end{array}$ & $\begin{array}{l}22.5 \\
1967\end{array}$ & $\begin{array}{l}21.7 . \\
1967\end{array}$ & $\begin{array}{l}14.8 . \\
1967\end{array}$ \\
\hline \multicolumn{9}{|l|}{ Algen: } \\
\hline Cyanophyta & 11. & 18 & 27 & 5 & 6 & 0,6 & 26 & 16 \\
\hline Chlorophyta & 1 & 2 & 1 & 0,6 & 0,5 & - & 13 & 8 \\
\hline Chrysophyceae & -- & - & - & - & - & - & 4 & - \\
\hline Diatomeae & 73 & 66 & 61 & 86 & 88 & 85 & 56 & 29 \\
\hline Peridineae & 12 & 12 & 10 & 6 & 3 & 0,4 & - & 35 \\
\hline \multicolumn{9}{|l|}{$\begin{array}{l}\text { Peridineae } \\
\text { Tiere: }\end{array}$} \\
\hline $\begin{array}{l}\text { Rotatoria, Phyllopoda, } \\
\text { Copepoda }\end{array}$ & 3 & 2 & 0,7 & 3 & 3 & 14 & 1 & 12 \\
\hline Total & 100 & 100 & 99,7 & 100,6 & 100,5 & 100 & 100 & 100 \\
\hline
\end{tabular}


sächlich Rädertiere (Keratella cochlearis, Polyarthra platyptera, Notholca longispina, Asplanchna priodonta) und einzelne Copepoden (Cyclops vicinus) und Phyllopoden (Daphnia longispina) wurden bei den Biomasseberechnungen auf Grund von teils Volumen- teils Stickstoffberechnungen so gut wie möglich berücksichtigt. Im Vergleich zu den Gesamtzahlen waren die Zooanteile klein; sie erreichten 0-15\%, durchschnittlich 5\% der Biomasse. Da das Volumen der grösseren Planktontiere kaum richtig zu berechnen ist, zählten wir zusätzlich fertig entwickelte Cyclops und Daphnien einzeln aus Zooplanktonproben heraus und ermittelten ihren durchschnittlichen Stickstoffgehalt. Für 11 Proben à 50 Cyclops vicinus erhielten wir pro Tier einen Durchschnittswert von $1,2 \mu \mathrm{gN}$, und 6 Proben à 50 Daphnia longispina ergaben $1,9 \mu \mathrm{gN}$ pro Organismus.

Kulturen: In einer zweiten Untersuchungsserie wollten wir die Beziehung zwischen der Biomasse und dem Gehalt an partikulärem Stickstoff unter genau definierten Bedingungen feststellen. Dazu verwendeten wir Oscillatoria rubescens, die mit den Kieselalgen zusammen eine der häufigsten Planktonalgen des Vierwaldstättersees ist. Ihre Biomasse (berechnet aus Schöpfproben) erreicht während der Herbst- und Wintermonate oft mehr als 50\%. Nachdem sie von STAUB [20] vor Jahren bakterienfrei kultiviert werden konnte, ist sie sozusagen die "Hausalge" unseres Institutes geworden. Ausserdem versuchten wir Tabellaria fenestrata, die zweite Massenform des Vierwaldstättersees zu kultivieren, jedoch ohne Erfolg. An ihrer Stelle entwickelte sich Synedru acus, eine ebenfalls zahlreiche Kieselalge.

Als Kulturmedium verwendeten wir für beide Formen autoklaviertes, filtriertes Seewasser (Vierwaldstättersee, Stelle Kreuztrichter, aus $75 \mathrm{~m}$ Tiefe, $24 \mu \mathrm{g} / \mathrm{PO}_{4}-\mathrm{P}$ ), ferner für Oscillatoria rubescens die für Blaualgen übliche Nährlösung Z8 [20] und für die Kieselalge Synedra acus die etwas modifizierte L-Lösung nach VoLLENWEIDER und SARACENi [24].

Die Kulturen wurden in Erlenmeyerkolben von 100 und $250 \mathrm{ml}$ Volumen bei $19-21^{\circ} \mathrm{C}$ und bei einer Beleuchtungsstärke von rund 500 Lux (Fluoreszensröhren Osram-L $20 \mathrm{~W} / 25$ weiss, die 12 Stunden pro Tag in Betrieb waren) gehalten.

Um das Trockengewicht bestimmen zu können, wurde der Niederschlag, der nach dem Autoklavieren der Nährlösungen entstand, steril wegfiltriert.

Schöpfproben: Während eines Jahres (Juli 1966 bis Juli 1967) analysierten und berechneten wir den Gehalt an partikulärem Stickstoff und die Biomasse in Schöpfproben, die monatlich in verschiedenen Tiefen des Vierwaldstättersees erhoben worden sind. Im Unterschied zu den Netz- und Kulturproben, wo wir stets mehrere Parallelbestimmungen durchgeführt haben, erfolgten diese Analysen nur einmal, das heisst, die Schöpfproben sind in der Art verarbeitet worden, wie es bei Seeuntersuchungen getan wird. Ferner wurde das Trockengewicht und die organische Trockensubstanz, abgesehen von wenigen Malen, aus zeitlichen und methodischen Gründen nicht bestimmt.

\section{Resultate und Diskussion}

Das Verhältnis von Trockengewicht bzw. aschenfreier Trockensubstanz zu partikulärem Stickstoff

Die Untersuchungsergebnisse über die Beziehung zwischen Trockengewicht $(T)$ bzw. aschenfreier Trockensubstanz $\left(T^{x}\right)$ und partikulärem Stickstoff $(\mathrm{p} . \mathrm{N})$ sind in 

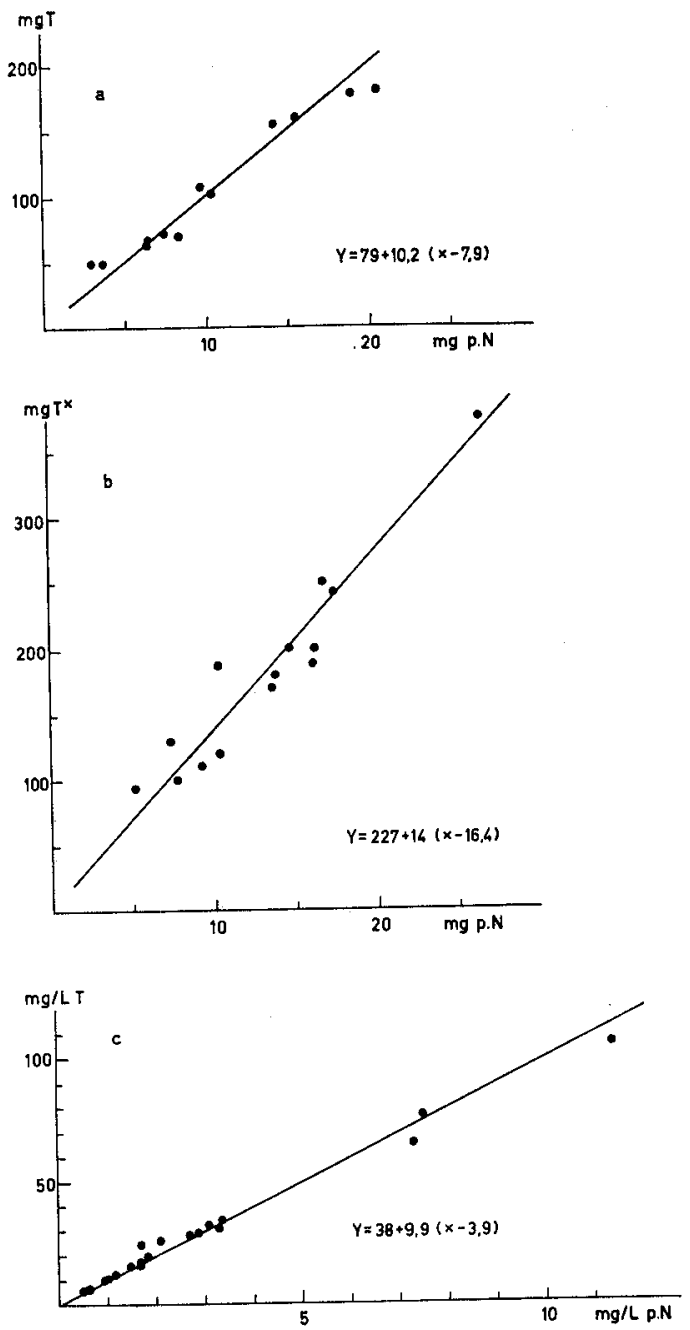

Abb. 1. Beziehung zwischen Trockengewicht (T) bzw. aschenfreier Trockensubstanz $\left(T^{x}\right)$ und partikulärem Stickstoff (p.N). a Netzplankton: Fraktion mit Oscillatoria rubescens; $b$ Netzplankton: Fraktion mit dem restlichen Plankton (hauptsächlich Kieselalgen); $c$ Kulturmaterial: Oscillatoria rubescens.

Form von Regressionsgeraden dargestellt (Abb. 1). Ferner ist das wichtigste Zahlenmaterial in den Tabellen 2-5 zusammengefasst ${ }^{1}$ ). Aus Abb. 1 geht hervor, dass der Anteil

1) Von Oscillatoria rubescens bestimmten wir meistens nur das Trockengewicht. Aus mehreren Veraschungen von Netz- und Kulturmaterialien erhielten wir für Oscillatoria rubescens einen Durchschnittswert von $95 \%$ aschenfreier Trockensubstanz, wobei eine vollständige Veraschung, das heisst bis zu weisser Asche, bis zu 12 Stunden benötigte. Lange Zeit blieb die Asche rötlich gefärbt, ein Stadium, in welchem der Aschengehalt 10\% betrug. Da der Aschengehalt klein und konstant war, verwendeten wir für die Abbildungen und die Berechnungen die gemessenen Trockengewichte der Oscillatoria-rubescens-Materialien. Für die Netzplanktonfraktionen mit den restlichen 
des partikulären Stickstoffs bezogen auf das Trockengewicht bzw. die aschenfreie Trokkensubstanz recht konstant ist. Besonders klein aber ist die Streuung für das bakterienfreie Kulturmaterial von Oscillatoria rubescens (vgl. Tab. 7). Jahreszeitliche Unterschiede, bedingt durch den Wechsel von Temperatur, Licht oder den Nährstoffgehalt des Wassers, konnten weder in den Netzmaterialien mit Oscillatoria rubescens noch in den gemischten Planktonproben festgestellt werden. STAUB [20] hingegen beobachtete in seinen Stickstoffanalysen von Oscillatoriamaterial aus dem Burgäschisee und Zürichsee eine deutliche Abnahme des Stickstoffgehalts gegen das Ende der Sommerstagnationszeit, parallel zur Abnahme des Nitratstickstoffs im See. Analog zu diesen Beobachtungen stellten wir die Abnahme des partikulären Stickstoffs in Kulturen von Oscillatoria rubescens fest, die mit filtriertem Seewasser als Nährmedium angesetzt wurden. In einer solchen Serie sank der Gehalt an partikulärem Stickstoff bis auf 4,8\% des Trockengewichtes (Tab. 4). Die Abnahme begann schon nach $10 \mathrm{Ta}-$ gen, wobei zugleich eine Verfärbung der weinroten Alge gegen Braun-Orange einsetzte. Vermutlich ist das auf eine Verarmung des Mediums an Nährstoffen zurückzuführen. (Nach 15 Tagen waren nur noch $0,01 \mu \mathrm{g} \mathrm{PO}_{4}-\mathrm{P}$ je $40 \mathrm{ml}$ nachweisbar.) Dies stimmt auch mit STAuB [20] und anderen Autoren (vgl. [20]) überein, die in Stickstoffmangelversuchen untere Grenzwerte von 5-6\% beobachtet haben. Im künstlichen Nährmedium Z8 konnte eine gute Beziehung zwischen Trockengewicht und Stickstoffgehalt festgestellt werden (vgl. Tab. 4). Der prozentuale Gehalt an partikulärem Stickstoff (rund 10\%) in Netz- und Kulturmaterial von Oscillatoria rubescens (Tab. 2 und 3) war stets höher als derjenige der Fraktion mit dem restlichen gemischten Netzplankton (rund $7 \%$ der aschenfreien Trockensubstanz, Tab. 4).

Tab. 2. Netzzüge (Vierwaldstättersee, Stelle Kreuztrichter), Fraktion mit Oscillatoria rubescens: Trockengewicht $(T)$, partikulärer Stickstoff (p.N), Biomasse $(B)$.

\begin{tabular}{|c|c|c|c|c|c|c|}
\hline $\begin{array}{l}\text { Datum } \\
\text { Probenahme }\end{array}$ & $\begin{array}{l}T \\
{[\mathrm{mg}]}\end{array}$ & $\begin{array}{l}\mathrm{p} \cdot \mathrm{N} \\
{[\mathrm{mg}] \% T}\end{array}$ & $\begin{array}{c}\mathrm{B} \\
{[\mathrm{mg}]}\end{array}$ & $T / \mathrm{p} . \mathrm{N}$ & $T / B$ & $B / \mathrm{p} . \mathrm{N}$ \\
\hline 24. 5. 1966 & 64 & $5,5 \quad(8,6)$ & 78 & 10,3 & 0,82 & 13 \\
\hline 23. 6.1966 & 51 & $3,7 \quad(7,3)$ & 62 & 13,5 & 0,75 & 17 \\
\hline 15. 8.1966 & 360 & $41 \quad(11,4)$ & 812 & 8,8 & 0,45 & 20 \\
\hline 14. 9.1966 & 106 & $9,7 \quad(9,2)$ & 173 & 11,3 & 0,65 & 18 \\
\hline 11.10 .1966 & 160 & $15,5 \quad(9,7)$ & 265 & 10,3 & 0,59 & 17 \\
\hline 7. 11.1966 & 102 & $10,4(10,2)$ & 173 & 9,6 & 0,59 & 17 \\
\hline 15.11 .1966 & 72 & $8,3(\mathbf{1 1}, 5)$ & 120 & 8,4 & 0,58 & 14 \\
\hline 7. 12.1966 & 178 & $19,0(10,7)$ & 475 & 7,3 & 0,38 & 25 \\
\hline 5. 1.1967 & 67 & $6,4 \quad(9,6)$ & 148 & 10,1 & 0,45 & 23 \\
\hline 30. 1.1967 & 72 & $7,4(10,3)$ & 117 & 9,5 & 0,58 & 16 \\
\hline $\begin{array}{ll}27 . & 2.1967 \\
22 . & 5.1967\end{array}$ & \multicolumn{6}{|c|}{ Oscillatoria rubescens war nicht gut vom Plankton zu trennen. } \\
\hline 21. 7. 1967 & 26 & $2,9(11,2)$ & 46 & 9,0 & 0,56 & 16 \\
\hline 14. 8.1967 & 155 & $14,2 \quad(9,2)$ & 259 & 11,3 & 0,62 & 18 \\
\hline
\end{tabular}

Planktonformen und für die Kulturen der Kieselalge Synedra acus hingegen war ein stark variierender eher niedriger Gehait an aschenfreier Trockensubstanz (35-74\%) charakteristisch, so dass es notwendig war, dieselbe jedesmal zu bestimmen und bei den Vergleichen und Berechnungen zu berücksichtigen (vgl. Tab. 3). 
Da die Proben der gemischten Netzplankter mit Ausnahme von drei Materialien mehr als $50 \%$ Kieselalgen aufzeigten (Tab. 1), wird angenommen, dass die wichtigsten Kieselalgen unserer Seen durchschnittlich weniger Stickstoff pro aschenfreie Trockensubstanz enthalten als die Blaualge Oscillatoria rubescens. Die Kulturproben der Kieselalge Synedra acus (Tab. 5) bestätigten diese Vermutung, indem drei Kulturserien Werte von 4-8\% partikulären Stickstoffs, bezogen auf die aschenfreie Trockensubstanz, ergaben.

Schon Birge und JUdAy [1] kamen auf entsprechende Resultate. Sie erhielten für 10 getestete Blaualgenmaterialien (Planktonproben), mit Ausnahme von einem tiefen Wert $(6,65 \%)$, Stickstoffanteile zwischen 8,66 und 10,6\%, für Kieselalgen einen solchen von $6,05 \%$, bezogen auf die aschenfreie Trockensubstanz. Bei GrIm [4] lesen wir 7 und $9 \%$ für die Blaualgengattungen Anabaena und Oscillatoria und 3,5 und 5\% (Mittelwerte von 25 bzw. 70 Bestimmungen) für Kieselalgen, diesmal bezogen auf das $\mathrm{SiO}_{2}$-freie Trockengewicht, das heisst, für die Bezugsgrösse des aschenfreien Trockengewichtes wären vor allem die prozentualen Kieselalgengehalte etwas höher. In LEwIN und Guillard ([7], S. 403) sind weitere von verschiedenen Autoren stammende prozentuale Stickstoffzahlen (bezogen auf das aschenfreie Trockengewicht) hauptsächlich mariner Kieselalgen aufgeführt. Der Gehalt an partikulärem Stickstoff aus Kulturmaterial variiert zwischen 5,6 und 10\%, bei einem Mittel von 7,4\%. Die Untersuchungen von vier marinen Kieselalgenarten aus Kulturen durch PARsovs et al. [15] erbrachten ähnliche Werte: 6,3-9,7\% (Mittel 7,2\%). Aus diesen wenigen Zahlen aus der Literatur geht ebenfalls hervor, dass die Blaualgen gegenüber den Kieselalgen einen erhöhten Gehalt an Kjeldahl-Stickstoff aufweisen.

Tab. 3. Netzzüge (Vierwaldstättersee, Stelle Kreuztrichter), Fraktion mit dem restlichen Plankton (hauptsächlich Diatomeen): Trockengewicht (T), Asche $(A)$, aschenfreie Trockensubstanz $\left(T^{x}\right)$, partikulärer Stickstoff (p.N), Biomasse $(B)$

\begin{tabular}{|c|c|c|c|c|c|c|c|c|}
\hline $\begin{array}{l}\text { Datum } \\
\text { Probenahme }\end{array}$ & $\begin{array}{l}T \\
{[\mathrm{mg}]}\end{array}$ & $\begin{array}{l}A \\
\% T\end{array}$ & $\begin{array}{l}T^{x} \\
{[\mathrm{mg}]}\end{array}$ & $\begin{array}{l}\text { p.N } \\
{[\mathrm{mg}] \% T^{x}}\end{array}$ & $\begin{array}{l}\mathrm{B} \\
{[\mathrm{mg}]}\end{array}$ & $T^{x} /$ p. N & $T^{x} / B$ & $B / \mathrm{p} \cdot \mathrm{N}$ \\
\hline 24. 5. 1966 & 2140 & 65 & 750 & $53 \quad(7,1)$ & 1800 & 14,1 & 0,42 & 34 \\
\hline 15. 8.1966 & 220 & 49 & 112 & $9,2(8,2)$ & 190 & 12,0 & 0,58 & 21 \\
\hline 14. 9.1966 & 260 & 28 & 187 & $10,3(5,5)$ & 374 & 18,4 & 0,56 & 36 \\
\hline 11. 10.1966 & 660 & 26 & 488 & $34,8(7,2)$ & 750 & 14,1 & 0,57 & 22 \\
\hline 7. 11. 1966 & 391 & 35 & 254 & $16,8(6,6)$ & 470 & 14,9 & 0,53 & 28 \\
\hline 7.12.1966 & 353 & 53 & 119 & $10,3(8,6)$ & 275 & 11,6 & 0,43 & 27 \\
\hline 5. 1.1967 & 485 & 59 & 199 & $14,7(7,4)$ & 376 & 13,6 & 0,53 & 26 \\
\hline 30. 1. 1967 & 352 & 45 & 192 & $16,1(8,4)$ & 306 & 11,8 & 0,61 & 19 \\
\hline 27. 2. 1967 & 328 & 47 & 174 & $13,6(7,8)$ & 302 & 12,5 & 0,57 & 22 \\
\hline 28. 3. 1967 & 173 & 46 & 93 & $5,2(5,6)$ & 149 & 18,2 & 0,63 & 29 \\
\hline 24. 4.1967 & 241 & 57 & 104 & $7,8(7,5)$ & 209 & 12,8 & 0,48 & 27 \\
\hline 22. 5.1967 & 432 & 57 & 186 & $13,8(7,4)$ & 445 & 13,0 & 0,40 & 32 \\
\hline 21. 7.1967 & 394 & 49 & 200 & $16,1(8,0)$ & 390 & 12,4 & 0,54 & 24 \\
\hline 14. 8.1967 & 196 & 34 & 129 & $7,3(5,7)$ & 170 & 17,8 & 0,76 & 23 \\
\hline
\end{tabular}

Halten wir die Resultate bezüglich Vergleich von Trockengewicht mit partikulärem Stickstoffgehalt nochmals fest. Mit Ausnahme von extremem Stickstoffmangel im Medium konnte eine relativ gute Übereinstimmung zwischen aschenfreier Trockensubstanz und partikulärem Stickstoff innerhalb der untersuchten Netzprobenserien 
und Kulturproben beobachtet werden. Die Grösse der Verhältniszahlen von Trockengewicht zu Stickstoff sind im wesentlichen von der Artenzusammensetzung des Materials abhängig. Unsere Werte ergaben ein durchschnittliches Verhältnis von 10 für Oscillatoria rubescens und ein solches von 14 für Kieselalgen. In der Literatur findet man einen allgemeinen Wert von 20 [16]. Solche allgemeinen Faktoren sind demnach mit Vorsicht zu gebrauchen.

Obgleich der partikuläre Stickstoff oft als zu stark variierende Zellkomponente betrachtet wird [22], das heisst dessen Gehalt zu sehr von den Spezies und von Umweltfaktoren wie Licht und Nährstoffangebot $[5,25]$ abhängig ist, kommen wir auf Grund unserer Resultate mit EinSELE [2], LUND [9, 10] und GRIM [4] zum Schluss, dass der Stickstoffgehalt eine relativ gute Grösse zur Erfassung der vorhandenen organischen, geformten Substanz in Kulturen und Netzzügen darstellt. Das Auseinandergehen der Werte verschiedener Autoren ist oft eher auf methodische Unterschiede zurückzuführen.

\section{Das Verhältnis von Trockengewicht bzw. aschenfreier Trockensubstanz zu Biomasse}

Aus Abb. 2 ist eine relativ gute Übereinstimmung des Trockengewichts bzw. der aschenfreien Trockensubstanz mit der berechneten Biomasse (vgl. auch Tab. 2-5) sowohl der Netzproben als des bis heute vorliegenden Kulturmaterials ersichtlich. Sie gibt uns die Gewissheit, dass bei der Berechnung der Volumina trotz vielen Mess- und Rechenoperationen tatsächlich einigermassen gesicherte Resultate erzielt werden können. Dies gilt auch für Formen wie Oscillatoria rubescens, die eine stark variierende Fadenlänge bzw. ein stark veränderliches Fadenvolumen aufweisen, so dass die Fäden von Probe zu Probe gemessen werden müssen. In unseren Seeproben bewegten sich die durchschnittlichen Fadenlängen zwischen 380 und $860 \mu$, in den Kulturen zwischen 150 und $930 \mu$.

Die aschenfreie Trockensubstanz aller berücksichtigten Proben variierte zwischen 30 und $70 \%$, bezogen auf das Zellvolumen. Der mittlere prozentuale Anteil betrug für die Netzfraktion mit Oscillatoria rubescens $60 \%$, bezogen auf die berechnete aschenfreie Trockensubstanz, für diejenige mit den restlichen Netzplanktern $57 \%$, für das Kulturmaterial von Oscillatoria rubescens $55 \%$ und für die Synedrakulturen $43 \%$. Eindeutig am tiefsten liegt der Anteil an aschenfreier Trockensubstanz für die Synedrakulturen, was - wie in der Literatur schon erwähnt wurde $[8,21]$ - darauf hinweist, dass für die Berechnung der Biomasse der Kieselalgen im Grunde genommen nicht das Zellvolumen, sondern, wegen der grossen Zellvakuolen, das Plasmavolumen berücksichtigt werden sollte. Um einen der Oscillatoria entsprechenden prozentualen Gehalt an aschenfreier Trockensubstanz zu erhalten, müssten die berechneten Zellvolumen bzw. die sogenannte Biomasse der Synedra acus um 20-30\% vermindert werden, das heisst, dass die Zellvakuolen ungefähr ein Drittel des Zellraumes einnehmen. Allerdings ist in den Netzfraktionen der Unterschied zwischen Zellvolumen und Zellplasma kaum spürbar. Möglicherweise werden in den gemischten Planktonproben Fehler, die bei der Berechnung der Volumina der verschiedenen Arten entstehen, wieder gegenseitig aufgehoben.

NALEWAJKo [13] verglich Trockengewicht, aschenfreie Trockensubstanz und Zellvolumen von 28 Algenarten aus Kulturen miteinander. Er erhielt mit einer Ausnahme 

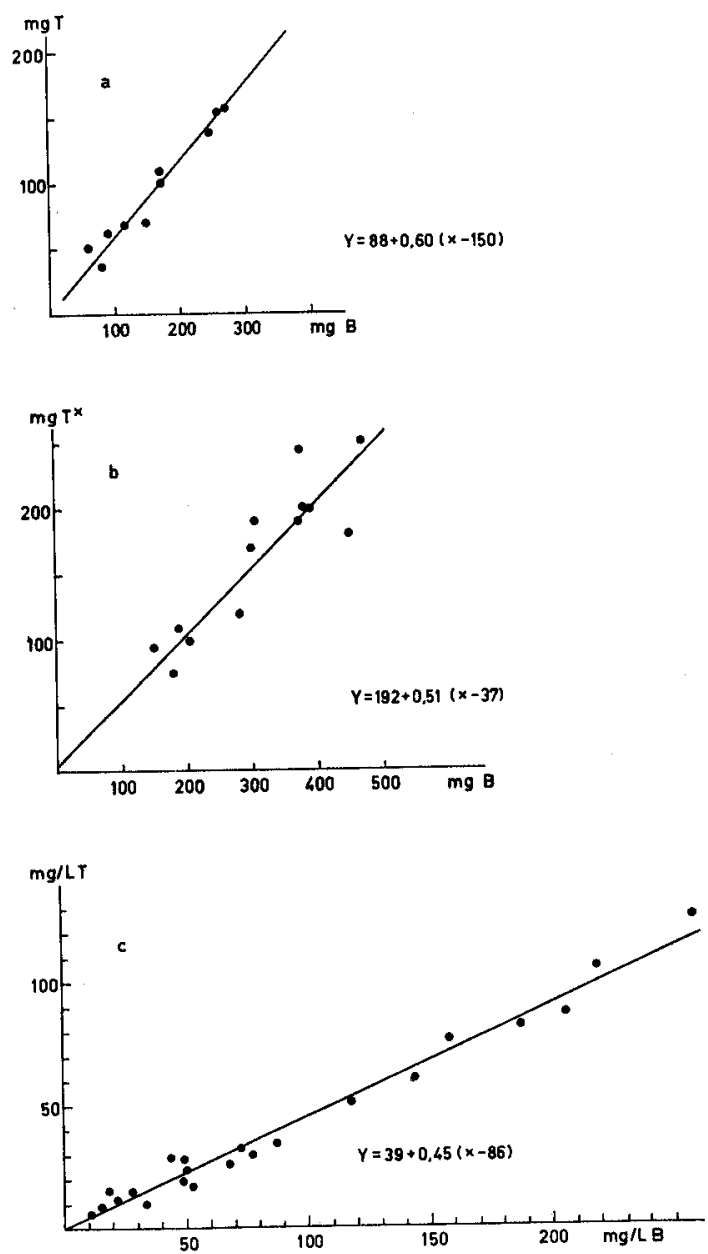

Abb. 2. Beziehung zwischen Trockengewicht $(T)$ bzw. aschenfreier Trockensubstanz $\left(T^{x}\right)$ und der Biomasse $(B)$. a Netzplankton: Fraktion mit Oscillatovia rubescens; $b$ Netzplankton: Fraktion mit dem restlichen Plankton (hauptsächlich Kieselalgen); $c$ Kulturmaterial: Oscillatoria rubescens.

10-50\% aschenfreie Trockensubstanz pro Zellvolumen, wobei die elf getesteten Kieselalgen nie über $30 \%$ enthalten. Gegenüber diesen Werten liegen unsere Prozentanteile etwas höher. Für Synedra acus, dem Namen nach die gleiche Kieselalgenart, die wir untersucht hatten, bestimmte er sogar nur $12 \%$ aschenfreie Trockensubstanz pro Biomasse (gegenüber durchschnittlich $43 \%$ in unserem Material).

Diese unterschiedlichen Werte sind vermutlich teils mit methodischen Faktoren (Bestimmung der aschenfreien Trockensubstanz und ganz besonders die Volumenberechnung), teils mit arteigenen Merkmalen der Organismen zu erklären. Auf jeden Fall zeigen diese Diskrepanzen einmal mehr, wie vorsichtig Werte aus der Literatur zu verwenden sind und wie schwierig es ist, solche Resultate miteinander zu vergleichen. 
Tab. 4. Kulturen von Oscillatoria rubescens, zwei Beispiele: Trockengewicht (T), partikulärer Stickstoff (p.N), Biomasse $(B)$ pro $150 \mathrm{ml}$ Nährmedium

\begin{tabular}{|c|c|c|c|c|c|c|c|}
\hline $\begin{array}{l}\text { Datum } \\
\text { Probenahme }\end{array}$ & $\begin{array}{l}T \\
{[\mathrm{mg}]}\end{array}$ & $\begin{array}{l}\mathrm{p} \cdot \mathrm{N} \\
{[\mathrm{mg}]}\end{array}$ & $\% T$ & $\begin{array}{l}B \\
{[\mathrm{mg}]}\end{array}$ & $T / \mathrm{p} . \mathrm{N}$ & $T / B$ & $B /$ p.N \\
\hline \multicolumn{8}{|c|}{ a) Filtriertes Seewasser } \\
\hline 19. 10.1966 & 1,5 & 0,16 & $(10,7)$ & 5,1 & 9,4 & 0,29 & 32 \\
\hline 25.10 .1966 & 4,5 & 0,50 & $(11,1)$ & 11,6 & 9,0 & 0,39 & 23 \\
\hline 28.10 .1966 & 9,1 & 0,60 & $(6,6)$ & 21,6 & 15,2 & 0,42 & 36 \\
\hline 31. 10. 1966 & 12,3 & 0,62 & $(5,1)$ & 28,1 & 19,8 & 0,43 & 45 \\
\hline 3.11. 1966 & 13,0 & 0,62 & $(4,8)$ & 30,9 & 21,0 & 0,43 & 60 \\
\hline \multicolumn{8}{|c|}{ b) Nährlösung Z 8} \\
\hline 23. 2. 1967 & 0,8 & - & - & 1,7 & - & 0,47 & - \\
\hline 27. 2.1967 & 1,8 & - & - & 3,3 & - & 0,53 & - \\
\hline 2. 3.1967 & 2,6 & 0,25 & $(9,6)$ & 7,9 & 10,0 & 0,33 & 30 \\
\hline 6.3 .1967 & 5,1 & 0,52 & $(10,2)$ & 13,1 & 9,1 & 0,39 & 26 \\
\hline 9. 3. 1967 & 7,7 & 0,75 & $(9,7)$ & 17,7 & 11,8 & 0,43 & 24 \\
\hline 13. 3. 1967 & 11,5 & 1,13 & $(9,8)$ & 23,7 & 10,1 & 0,48 & 21 \\
\hline 1.6. 3. 1967 & 15,8 & 1,71 & $(10,8)$ & 32,8 & 9,2 & 0,48 & 19 \\
\hline 20.3 .1967 & 19,3 & 2,22 & $(11,5)$ & 38,2 & 8,6 & 0,51 & 17 \\
\hline 23. 3. 1967 & 18,9 & 1,86 & $(9,8)$ & 39,4 & 10,2 & 0,48 & 21 \\
\hline
\end{tabular}

Abschliessend kann man jedoch zu diesem Abschnitt wohl sagen, dass durch die Vergleiche des berechneten Zellvolumens mit der Bezugsgrösse der organischen Trokkensubstanz die mit Recht bezweifelte sogenannte Biomasse klarer erfasst wird, das heisst, innerhalb tragbarer Fehlergrenzen kann von der berechneten Biomasse auf die organische Trockensubstanz und umgekehrt von der aschenfreien Trockensubstanz auf die Biomasse von Netzproben und Kulturmaterial geschlossen werden.

Tab. 5. Kulturen von Synedra acus, Material aus zwei Serien: 'Trockengewicht (T), Asche $(A)$, aschenfreie Trockensubstanz $\left(T^{x}\right)$, partikulärer Stickstoff (p.N), Biomasse $(B)$.

\begin{tabular}{|c|c|c|c|c|c|c|c|}
\hline $\begin{array}{l}T \\
{[\mathrm{mg}]}\end{array}$ & $\begin{array}{l}A \\
\% T\end{array}$ & $\begin{array}{l}T^{x} \\
{[\mathrm{mg}]}\end{array}$ & $\begin{array}{l}\mathrm{p} . \mathrm{N} \\
{[\mathrm{mg}] \% T^{x}}\end{array}$ & $\begin{array}{l}\mathrm{B} \\
{[\mathrm{mg}]}\end{array}$ & $T^{x} / \mathrm{p} . \mathrm{N}$ & $T^{x} / \mathrm{B}$ & $B /$ p.N \\
\hline \multicolumn{8}{|c|}{ 10.-17. 10.1967 (17. 10. Erntetag) } \\
\hline 32,7 & $\left.65^{1}\right)$ & 11,5 & $0,90 \quad(7,8) \quad 3$ & 39,3 & 12,7 & 0,29 & 44 \\
\hline 31,3 & & 11,0 & $0,85(7,7)$ & 37,3 & 12,9 & 0,29 & 44 \\
\hline 41,3 & & 11,5 & $0,87(7,6) \quad 4$ & 42,6 & 16,6 & 0,34 & 49 \\
\hline 39,9 & & 14,0 & $0,92(6,6) \quad 4$ & 41,4 & 15,2 & 0,34 & 45 \\
\hline 42,6 & & 15,0 & $0,98(6,5)$ & 47,4 & 15,2 & 0,32 & 48 \\
\hline \multicolumn{8}{|c|}{ 17.-30.11. 1967 (30.11. Erntetag) } \\
\hline 99,7 & 62 & 37,5 & $\left.1,70^{\mathbf{1}}\right) \quad(4,9)$ & 76,0 & 22,1 & 0,49 & 45 \\
\hline 104,2 & 74 & 27,4 & & 68,0 & 16,1 & 0,40 & 40 \\
\hline 100,7 & 62 & 38,7 & & 79,0 & 22,8 & 0,49 & 46 \\
\hline 105,2 & 69 & 32,7 & & 75,0 & 19,5 & 0,44 & 44 \\
\hline 106,0 & 67 & 35,3 & & 74,0 & 20,8 & 0,48 & 44 \\
\hline
\end{tabular}




\section{Das Verhältnis von Biomasse zu partikulärem Stickstoff}

In diesem Abschnitt kommen wir nun zur Besprechung der Resultate, die die einleitenden Fragen über den Zusammenhang von Biomasse und partikulärem Stickstoff beantworten sollen.

Beginnen wir mit dem Vergleich der Biomasse mit dem Gehalt an partikulärem Stickstoff der Netz- und Kulturmaterialien. Die Regressionsgeraden in Abbildung 3 zeigen, dass innerhalb der berücksichtigten Proben jeweils eine gute Beziehung zwischen den beiden Komponenten besteht. Die durchschnittlichen Verhältniszahlen von Biomasse $(B)$ zu partikulärem Stickstoff (p.N) (Tab. 7) der verschiedenen Testmaterialien jedoch weisen grosse Unterschiede auf; sie variieren zwischen 16,5 und 43,7.
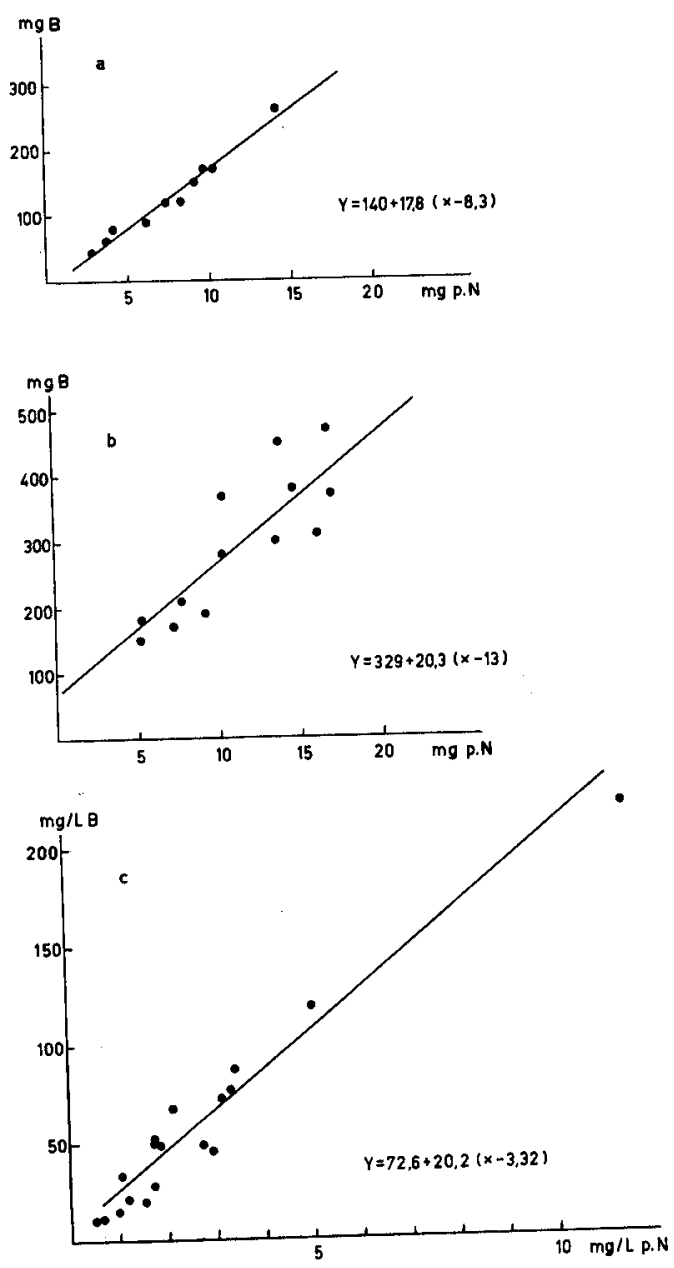

Abb. 3. Beziehung zwischen Biomasse $(B)$ und partikulärem Stickstoff (p.N). a Netzplankton: Fraktion mit Oscillatoria nubescens; $b$ Netzplankton: Fraktion mit dem restlichen Plankton (hauptsächlich Kieselalgen); c Kulturmaterial: Oscillatoria rubescens. 
Tab. 6. Schöpfproben (Vierwaldstättersee, Stelle Kreuztrichter): Biomasse des Phytoplanktons $B(P)$ und der Bakterien $B(B)$ im Vergleich zum Gehalt des partikulären Stickstoffs (p.N) in $\mathrm{mg} / \mathrm{l}$. Zahlen in Klammern bedeuten prozentuale Anteile, bezogen auf die Biomasse des Phytoplanktons.

\begin{tabular}{|c|c|c|c|c|c|c|c|}
\hline $\begin{array}{l}\text { Datum } \\
\text { Probenahme }\end{array}$ & $\begin{array}{l}\text { Tiefe } \\
{[\mathrm{m}]}\end{array}$ & $B(P)$ & $\left.B(B)^{\mathbf{1}}\right)$ & $B(P+B)$ & p.N & $B(P) / \mathrm{p} . \mathrm{N}$ & $B(P+B) / \mathrm{p} \cdot \mathrm{N}$ \\
\hline \multirow[t]{3}{*}{ 27. 2. 1967} & 0 & 0,6 & $0,08(13)$ & 0,68 & 0,03 & 20 & 23 \\
\hline & 5 & 0,9 & $0,09(10)$ & 0,99 & 0,08 & 30 & 33 \\
\hline & 15 & 1,1 & $0,07 \quad(6)$ & 1,17 & 0,03 & 55 & 59 \\
\hline \multirow[t]{4}{*}{24.4 .1967} & 0 & 1,3 & $0,55(42)$ & 1,85 & 0,10 & 13 & 19 \\
\hline & 2,5 & 0,9 & $0,54(60)$ & 1,45 & 0,08 & 11 & 18 \\
\hline & 5 & 1,0 & $0,61(61)$ & 1,61 & 0,11 & 9 & 15 \\
\hline & 15 & 1,4 & $0,56(40)$ & 1,96 & 0,10 & 14 & 20 \\
\hline \multicolumn{8}{|c|}{ 1) Werte von ScHEGG (1967) } \\
\hline
\end{tabular}

Den kleinsten Anteil an Stickstoff pro Biomasse wurde, wie zu erwarten war, in den Synedrakulturen bestimmt (2,8\%). Die gemischten Planktonproben ergaben einen Anteil von 3,7\%, Oscillatoria rubescens aus Kulturen enthielt 4,9\% und diejenigen aus den Planktonzügen knapp 6,1\%.

Auch in diesen Prozentzahlen kommt, wie weiter oben schon diskutiert wurde, einerseits das zu grosse Zellvolumen im Vergleich zur organischen Substanz des Zellraumes (Zellvakuolen), andererseits die Tatsache, dass Kieselalgen weniger Stickstoff pro organische Trockensubstanz enthalten als die Blaualge Oscillatoria rubescens, zum Ausdruck. Würden wir zum Beispiel die berechnete Biomasse der Synedrakulturen wiederum um etwa $30 \%$ (Zellvolumen) vermindern, so würde der prozentuale Anteil Stickstoff, bezogen auf die umgerechnete Biomasse, $4 \%$ ausmachen. Dieser Wert wäre immer noch um ein Drittel kleiner als der entsprechende Stickstoffgehalt der Oscillatoria rubescens aus Netzproben.

Kommen wir schliesslich auf die Resultate der untersuchten Schöpfproben zu sprechen. Die Werte des partikulären Stickstoffs und der Biomasse der monatlichen Schöpfprobenserien sind in Abb. 4 unter Berücksichtigung der Stichproben von 0-50 m aufgezeichnet. In grossen $Z$ ïgen beurteilt, verlaufen die monatlichen Kurven der beiden Komponenten sich deckend oder meistens parallel zueinander. Somit geben beide Kurven ein ähnliches Bild von der Verteilung der organisch geformten Substanz in Raum und Zeit des Sees.

Bei genauerem Vergleich der einzelnen Probeserien untereinander können wir besonders in jenen vom 18. 7. 1966 und vom 7.11. 1966 variierende Differenzflächen feststellen. Aber auch innerhalb von ein und derselben Kurve treten in einzelnen Serien (12.9. 1966 und 22. 5. 1967) Unterschiede auf, so dass das Differenzenband Biomasse-partikulärer Stickstoff eine ungleichmässige Breite aufweist (Abb. 4).

Das Verhältnis $B / \mathrm{p} . \mathrm{N}$ war denn auch nicht mehr so konstant, wie wir es von den Netz- und Kulturmaterialien her gewohnt gewesen waren. Wohl sind die Verhältnisse innerhalb einer Probeserie mit wenigen Ausnahmen konstant, können aber von Mal zu Mal stark ändern (Abb. 5, Tab. 7). Der Mittelwert der Verhältniszahlen von Biomasse zu Stickstoff von gut 80 Proben der Tiefenstufen zwischen 0 und $20 \mathrm{~m}$ (vgl. Abb. 4) betrug 22,1. Er lag an sich in der Nähe der ermittelten Faktoren der anderen geprüften 

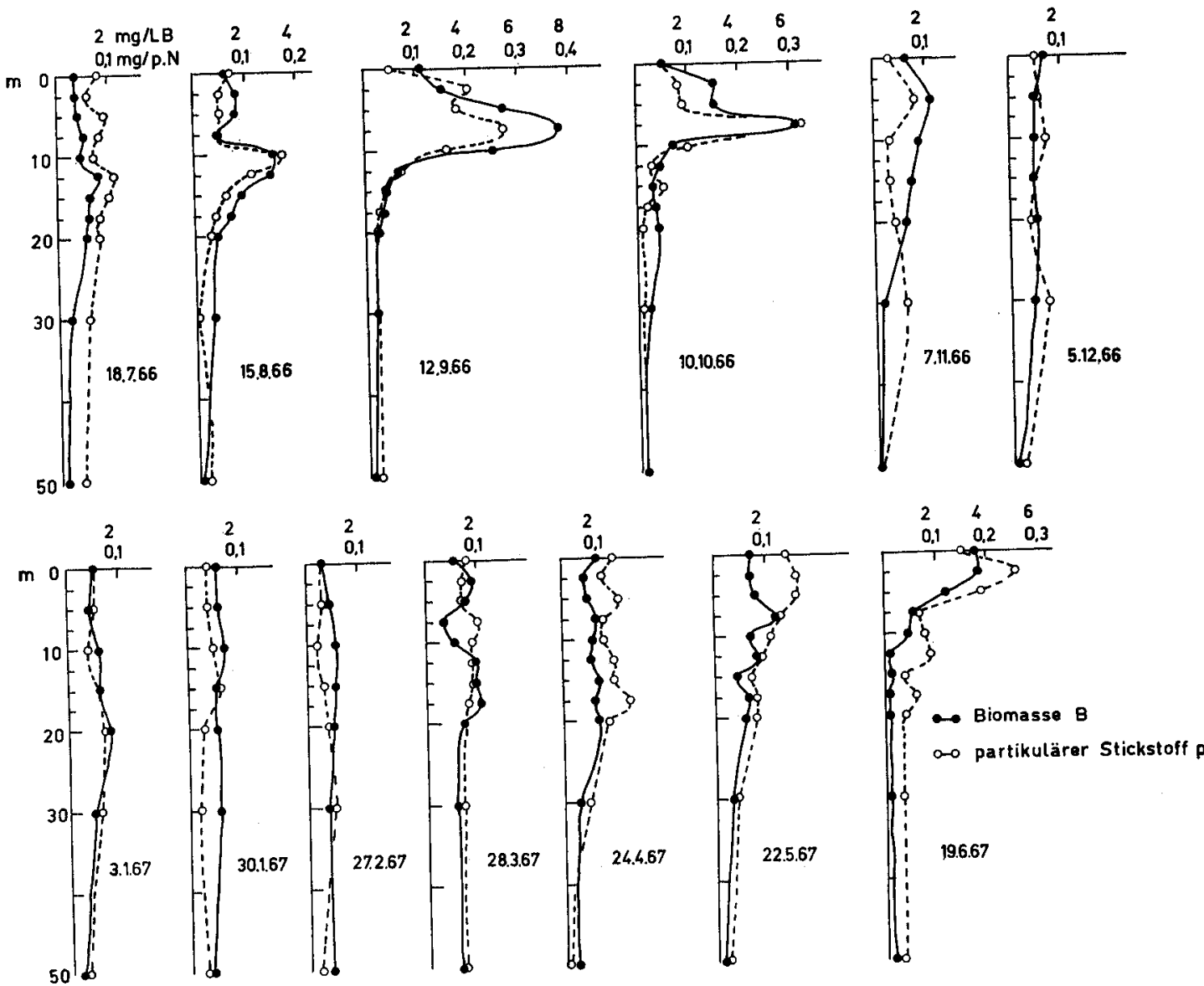

Abb. 4. Schöpfproben (Vierwaldstättersee, Stelle Kreuztrichter): Biomasse $(B)$ des Phytoplanktons und Gehalt an partikulärem Stickstoff (p.N).

Materialien (mit Ausnahme der Synedra-acus-Kulturen), während die Streuung gegenüber Netz- und Kulturproben viel grösser ist (Tab. 7).

Woher rühren die unterschiedlichen Verhältniszahlen $B / p . N$ der Schöpfproben? Folgende drei möglichen Ursachen seien erwähnt:

1. Wie schon anhand der Netz- und Kulturmaterialien gezeigt wurde, kann der Quotient Biomasse/partikulärer Stickstoff je nach Art der Alge bzw. je nach Zusammensetzung des Planktons ändern.

Die drei höchsten clurchschnittlichen Verhältniszahlen $B / \mathrm{p} . \mathrm{N}$ von $0-20 \mathrm{~m}$ Tiefe erhielten wir mit 45, 30, 35 in den Monaten November 1966, Januar und Februar 1967 (vgl. Abb. 4). Im Monat November machte neben der Oscillatoria rubescens (etwa 50\%) die Peridinee Ceratium hirundinella etwa $25 \%$ der Biomasse aus. Möglicherweise wird das Volumen dieser komplizierten Form stets zu gross berechnet. Die hohen Faktoren vom Januar und Februar können eindeutig mit der grossen Biomasse von Kieselalgen (je $50 \%$ ) gedeutet werden. 


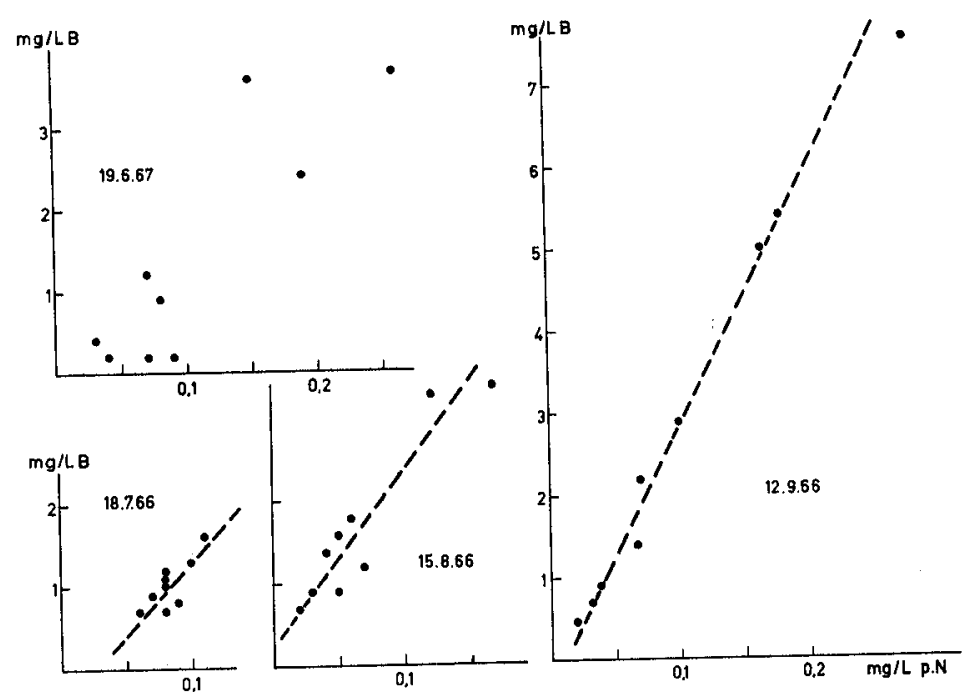

Abb. 5. Einzelne Schöpfprobenserien (Vierwaldstättersee, Stelle Kreuztrichter): Beziehung zwischen der Biomasse $(B)$ des Phytoplanktons und dem Gehalt an partikulärem Stickstoff (p.N).

Hohe prozentuale Biomasseanteile an Kieselalgen (mit 40 bis über $75 \%$ ) fallen ferner in den Monaten März bis Juni 1967 auf. Bemerkenswert in diesen Monaten sind aber die Verhältniszahlen $B /$ p.N, die entgegen den Resultaten aus Kultur- und Netzproben tiefe Werte aufweisen. Damit kommen wir zur zweiten Ursache, die die Unterschiede der Verhältniszahlen $B / \mathrm{p} . \mathrm{N}$ der Schöpfproben grundlegend beeinflussen kann.

2. Verhältniszahlen von Biomasse zu partikulärem Stickstoff, die wesentlich tiefer liegen als der durchschnittliche Faktor 22 der Schöpfproben, können dadurch erklärt werden, dass die Bestimmung des partikulären Stickstoffs die gesamte geformte Substanz, eigentlich das gesamte Seston erfasst, also auch die Bakterien, den Detritus, die gerade in Schöpfproben in grosser Menge beobachtet werden können. Die berechnete Biomasse hingegen gibt nur den Gehalt der geformten organischen Substanz der ausgezählten Formen wider.

Wie gross der Anteil der Biomasse der Bakterien im Vergleich zur Phytoplanktonbiomasse im Vierwaldstättersee sein kann, gibt Tabelle 6 Auskunft. Die Bakterienzahlen stammen von ScHEGG (1967), der ihre Biomasse auf Grund von Direktzählungen der Bakterien ermittelt hat. Die beiden Beispiele zeigen je eine Probeserie mit kleinem (27.2. 1967) und grossem Bakterienanteil (24. 4. 1967). Wir sehen, wie die auffallend tiefen Verhältniszahlen $B / \mathrm{p} . \mathrm{N}$ bei Berücksichtigung der Bakterien sich entsprechend verändern. Auch in den Monaten Mai und Juni 1967, also zur Zeit der starken Kieselalgenentwicklung, war der Anteil der Bakterien hoch. Sie hatten demnach zu einem wesentlichen Teil die oben erwähnten tiefen Werte der Verhältniszahlen $B /$ p. N in diesen Monaten bewirkt.

3. Die grössten Verhältniszahlen $B / \mathrm{p} . \mathrm{N}$ stellten wir in den Tiefenproben in 50-100 m Tiefe fest, wo die Faktoren 50, 60, ja 80 betrugen. Ursache dieser extremen Zahlen sind einerseits in den niederen Werten des partikulären Stickstoffs unterhalb 
Tab. 7. Verhältniszahlen von Trockensubstanz $(T)$ bzw. aschenfreier Trockensubstanz zu partikulärem Stickstoff (p.N), von Trockensubstanz bzw. aschenfreier Trockensubstanz zu Biomasse $(B)$ und von Biomasse zu partikulärem Stickstoff: Mittelwerte und Streuungen. Zahlen in Klammern bedeuten Anzahl der gemittelten Werte.

\begin{tabular}{|c|c|c|c|}
\hline Untersuchtes Material & $T / \mathrm{p} . \mathrm{N}$ & $T / B$ & $B / \mathrm{p} . \mathrm{N}$ \\
\hline $\begin{array}{l}\text { Netzproben: } \\
\text { Oscillatoria rubescens (12) } \\
\text { Restliches Phytoplankton (14) }{ }^{\mathbf{1}} \text { ) }\end{array}$ & $\begin{array}{l}10,3 \pm 1,95 \\
14,1 \pm 2,41\end{array}$ & $\begin{array}{l}0,57 \pm 0,11 \\
0,57 \pm 0,09\end{array}$ & $\begin{array}{l}16,5 \pm 1,75 \\
27,3 \pm 5,20\end{array}$ \\
\hline $\begin{array}{l}\text { Algenkulturen: } \\
\text { Oscillatoria rubescens (24) } \\
\left.\text { Synedra acus }(\mathbf{1 5})^{\mathbf{1}}\right)\end{array}$ & $\begin{array}{l}10,2 \pm 0,83 \\
17,9 \pm 2,82\end{array}$ & $\begin{array}{l}0,52 \pm 0,06 \\
0,43 \pm 0,11\end{array}$ & $\begin{array}{l}20,3 \pm 3,13 \\
43,7 \pm 3,28\end{array}$ \\
\hline $\begin{array}{l}\text { Schöpfproben (unfiltriertes Seewasser): } \\
\text { Monatliche Proben aus verschiedenen } \\
\text { Tiefen von } 0 \text { bis } 20 \mathrm{~m}(83) \\
\end{array}$ & - & - & $22,7 \pm 10,6$ \\
\hline $\begin{array}{l}\text { Einzelne Probeserien: } \\
\text { 18.7.1966(9) } \\
\text { 12.9.1966(9) } \\
\text { 19.6.1967 (9) }\end{array}$ & $\begin{array}{l}- \\
- \\
-\end{array}$ & - & $\begin{array}{l}13,2 \pm 2,3 \\
26,4 \pm 4,1 \\
11,1 \pm 7,3\end{array}$ \\
\hline
\end{tabular}

der erfassbaren Genauigkeit von 0,01-0,02 mg/l zu suchen, andererseits darin, dass in den Tiefenproben oft auch leere und tote Zellen beim Auszählen berücksichtigt werden.

\section{Schlussfolgerungen}

Die untersuchten Materialien (Netzprobenfraktionen, Kulturen von Oscillatoria rubescens und Synedra acus und Schöpfproben) ergaben im wesentlichen folgende Resultate:

1. Trockengewicht bzw. aschenfreies Trockengewicht, partikulärer Stickstoff und Biomasse zeigen innerhalb der Netzfraktionen und Kulturproben eine gute Übereinstimmung, was in den Figuren 1-3 zum Ausdruck kommt. Je nach Algenart und -zusammensetzung variieren die gegenseitigen Verhältniszahlen von Trockengewicht, Stickstoff und Biomasse (Tab. 7). Diese unterschiedlichen Verhältniszahlen sind bedingt einerseits durch den geringeren Stickstoffgehalt der Kieselalgenzellen pro aschenfreie Trockensubstanz im Vergleich zu Blaualgen, andererseits durch die relativ grossen Zellvakuolen der Kieselalgen und die dadurch zu hoch berechneten Biomassewerte.

2. Für die Probeserien der Schöpfproben sind die Verhältniszahlen von Biomasse zu Stickstoff innerhalb einer Probeserie meistens konstant, können aber von Mal zu Mal stark ändern (Tab. 7, Abb. 4 und 5). Ursachen dafür liegen hauptsächlich darin, dass durch die indirekte Bestimmung des partikulären Stickstoffs und die Filtration des Seewassers durch ein Filter mit einer Porenweite von nur $0,45 \mu$ der N-Gehalt sozusagen der gesamten organisch geformten Substanz erfasst wird, während die Biomassebestimmung nur einen Wert der Gesamtheit der gezählten Organismen ergibt. 
3. Als gemeinsames Resultat für alle untersuchten Materialien kann aufgeführt werden, dass eine Beziehung zwischen Biomasse und partikulärem Stickstoffgehalt besteht, die innerhalb eines genau erfassbaren und definierbaren Materials bis auf wenige Ausnahmefälle recht konstant ist, aber je nach Beschaffenheit und Herkunft des Materials variiert. Daher muss die Beziehung der beiden diskutierten Komponenten jeweils zuerst festgestellt werden, das heisst, es genügt nicht, eine Komponente allein zu bestimmen, um daraus den Gehalt der anderen sicher zu berechnen, sondern es müssen jeweils auch die entsprechenden Kontroll- oder Bezugsgrössen mindestens einmal bestimmt werden, um gleichsam den «Eichwert» der Beziehungen zu ermitteln.

4. Auf Grund der Untersuchungen und den daraus erzielten Resultaten würden wir als praktische Anwendung der Stickstoffmethode bei Seeuntersuchungen, speziell bei routinemässigen Überwachungsprogrammen zur Erfassung der momentanen geformten organischen Substanz folgende Untersuchungsweise vorschlagen: Die Bestimmung des partikulären Stickstoffs in verschiedenen Tiefenstufen und die Berechnung der Biomasse von mindestens einer oder von zwei Mischproben (das heisst Proben, die aus verschiedenen Tiefen im gewogenen Mittel gemischt werden) je nach Schichtung des Sees, so dass ein durchschnittlicher Faktor zur Berechnung des Planktons zur Zeit der Probenahme erfasst werden kann. Damit, glauben wir, könnte im Zusammenhang mit Problemen des Gewässerschutzes rasch ein Bild vom biologischen Zustand des Sees gewonnen werden.

\section{Zusammenfassung}

Im Zusammenhang mit der Verbesserung der Stickstoffmethode nach Kjeldahl durch Schmid [18] wurde die Übereinstimmung des partikulär gebundenen Stickstoffs (p.N) mit der aus Individuenzahl und Zellvolumen berechneten Biomasse $(B)$ des Phytoplanktons überprüft. Ausser p. $N^{\prime}$ und $B$ wurde zum Teil auch die Trockensubstanz bzw. die aschenfreie Trockensubstanz gleichsam als Kontroll- und Bezugsgrösse bestimmt.

Untersucht wurde das Phytoplankton aus Netzproben und Schöpfproben des Vierwaldstättersees im Ablauf eines Jahres; ferner das Kulturmaterial der Planktonalgen Oscillatoria rubescens und Synedra acus.

Sowohl Netzproben als Algen aus Kulturen zeigen eine klare Beziehung 1. zwischen aschenfreier Trockensubstanz und partikulärem Stickstoff, 2. zwischen aschenfreier Trockensubstanz und Biomasse und 3. zwischen Biomasse und partikulärem Stickstoff. Die Verhältniszahlen der verglichenen Komponenten variieren je nach Algenart und Zusammensetzung des Planktons. Der prozentuale p. N-Gehalt (bezogen auf die aschenfreie Trockensubstanz) der Oscillatoria rubescens beträgt rund $10 \%$, derjenige der Kieselalgen rund $7 \%$.

Das Verhältnis von $B$ zu p.N der Schöpfproben hingegen variierte von Probeserie zu Probeserie. Innerhalb der einzelnen Probeserien jedoch ist das Verhältnis von $B$ zu p. N relativ konstant. Es werden Gründe diskutiert, die diese Unterschiede bedingen.

Um das mühsame Auszählen der Planktonorganismen und die Berechnung der Biomasse bei Seeuntersuchungen, speziell bei routinemässigen Überwachungsprogrammen, teilweise $z u$ umgehen, wird eine Untersuchungsweise vorgeschlagen, die es 
ermöglicht, die organisch geformte Substanz und zugleich die Zusammensetzung des Planktons mit verhältnismässig geringem Arbeitsaufwand zu erfassen.

Tch möchte Herrn M. Schmid, dem ehemaligen Chemiker der Limnologischen Abteilung der EAWAG, meinen herzlichen Dank aussprechen für Diskussion und Gespräch, besonders möchte ich ihm für die zahlreichen, sorgfältig durchgeführten Stickstoffbestimmungen danken. Ohne dieselben hätten die Vergleiche zwischen Stickstoff und Biomasse nicht angestellt werden können. Herrn H. Bachmann gilt mein Dank für viele Hilfeleistungen, speziell aber für die zuverlässige Durchführung der Kulturversuche. Frau P. Weber danke ich herzlich für die stets mit guter Laune bewältigte Arbeit des Auszählens der Schöpfproben.

\section{SUMMARY}

In connection with the improvement of the nitrogen method by ScHMid (18), the agreement of particulate nitrogen with the biomass (determined by the individuals counted and the cell volume calculated) of the phytoplankton was tested. For control and reference the dry weight and the ashfree dry weight or organic dry weight respectively were determined.

Three types of material were investigated: Phytoplankton from net samples and samples of unfiltered lakewater which were taken monthly during a year from the Lake of Lucerne and algal material from cultures of the two common plankton algae Oscillatoria rubescens and Synedra acus.

Within the net fractions and culture samples a good agreement is observed 1) between ash-free dry weight and particulate nitrogen, 2) between ash-free dry weight and biomass, 3) between biomass and particulate nitrogen.

The proportional figures of the compared components vary depending on the alga species and the composition of the plankton. The content of particulate ritrogen (per ash-free dry weight) amounted to about $10 \%$ for Oscillatoria rubescens and about $7 \%$ for diatoms. The ratio of biomass to particulate nitrogen of the samples of unfiltered lakewater may alter from one sample series to another. Within the single sample series however, the relationship of the biomass to the particulate nitrogen is generally constant. Reasons for these differences are discussed.

On the basis of the investigations and the results obtained therefrom, a combined method applying the determination of the particulate nitrogen and the biomass is suggested. This method allows to reduce the time consuming calculation of the biomass in lake investigations, more particularly in routine survey programs. On the other hand it enables to determine both the organic substance formed at the moment and the composition of the plankton in relatively short time.

\section{J.ITERATURVERZEICHNIS}

1] Brrge E. A., JUdAY C., The Inland Lakes of Wisconsin. The Plankton I. Its Quantity and Chemical Composition, Wisconsin Geological and Natural History Survey, Bull. Nr. 64 scientific Series Nr. 13, 222 (1922).

[2] EINSELE W., Die Umsetzung von zugefïhrtem, anorganischem Phosphat im eutrophen See und ihve Rückwirkung auf seinen Gesamthaushalt, Z. Fisch. 39 (3), 407-488 (1941).

[3] Gessner F., Hydrobotanik II (Berlin 1959), S. $489 \mathrm{ff}$.

[4] Grim J., Der Phosphor und die pflanzliche Produktion im Bodensee, GWF 108 (44), 1261-1271 (1967).

[5] HaRvey H. W., Synthesis of Organic Nitrogen and Chlorophyll by Nitzschia Closterium, J. Mar. biol. Ass. U. K. 37, 477-478 (1953).

[6] Krishnam URThy K., Nitrogen and Phosphorus in Plankton, Hydrobiologia 30, 273-280 (1967).

[7] Lewin J. C., Guillard R. R. L., Diatoms, A. Rev. Microbiol. 17, 373-414 (1963).

[8] Lohmann H., Untersuchungen zur Feststellung des vollständigen Gehaltes des Meeres an Plankton, Wiss. Meeresunters. 10, 131-370 (1908).

[9] Lund J. W. G., Primary Productivity and Periodicity of Phytoplankton, Ver. int. Verein. theor. angew. Limnol. 15, 37-56 (1964).

[10] Lund J. W. G., The Ecology of the Freshwater Phytoplankton, Biol. Rev. 40, 231-293 (1965). 
[11] Mackenthun K. M., Nitrogen and Phosphorus in Water. An Annotated Selected Bibliography of their Biological Effects (Public Health Service Publ. Nr. 1305, Washington, D. C. 1965).

12] Mullin M. M., Sloan P. R., Eppley R. W., Relationship Between Carbon Content, Cell Volume and Area in Phytoplankton, Limnol. Oceanogr. 11, 307-311 (1966).

[13] Nalewajko C., Dry Weight, Ash and Volume Data for Some Freshwater Planktonic Algae, J. Fish. Res. Bd. Can. 23, 1285-1288 (1966).

[14] PaAsche E., On the Relationship Between Primary Production and Standing Stock of Phytoplankton, J. Cons. perm. Int. Explor. Mer 26, 33-48 (1960).

15] Parsons T. R., Stephens K., Strickland J. D. H., On the Chemical Composition of Eleven Species of Marine Phytoplankters, J. Fish. Res. Bd. Can. 18, 1001-1016 (1961)

[16] Ruttner F., Grundriss der Limnologie, (Berlin 1962), S. 186 und 185.

[17] SchEGg E., Die limnische Produktion unter besonderer Berichschiedenem Trophiegrad, DiplomMessungen während der Frïhjahrsentwicklung in Seen

[18] Schмid M., Die Bestimmung kleiner Mengen von organischem Stickstoff im Wasser von Binnenseen, Schweiz. Z. Hydrol. 30, 244-266 (1968).

[19] Schmid M., АмвÜнLL H., Die Bestimmung geringster Mengen von Gesamtphosphor im Wasser von Binnenseen, Schweiz. Z. Hydrol. 27, 184-192 (1965).

20] STAuB R., Ernährungsphysiologisch-autökologische Untersuchungen an der planktischen Blaualge Oscillatoria rubescens DC, Schweiz. Z. Hydrol. 23, 82-198 (1961)

[21] Strathmann R. R., Estimating the Organic Carbon Content of Phytoplankton from Cell Volume or Plasma Volume, Limnol. Oceanogr. 12, 411-418 (1967).

22] Strickland J. D. H., Production of Organic Matter in the Primary Stages of the Marine Food Chain, in: RILEY J. P., SkirRow G., Chemical Oceanography I, (London and New York 1965), S. $477-610$.

[23] UTERмӧнL H., Zur Vevvollhommnung der quantitativen Phytoplankton-Methodik, Mitt. int. Verein. theor, angew. Limnol. Nr. 9 (1958).

[24] VollenweIder R. A., SaRACEnI C., Un muovo terreno nutrizio per la coltivazione di alghe planctoniche d'acqua dolce, Memorie Ist. ital. Idrobiol. 17, 215-221 (1964).

[25] Yentsch C. S., Vaccaro R. F., Phytoplankton Nitrogen in the Octans, Limnol. Oceanogr. 3 , 443-448 (1958).

Anschrift des Verfassers:

Frau Dr. Marianne Bosli-Pavoni, Eidg. Anstalt für Wasserversorgung, Abwasserreinigung und Gewässerschutz an der Eidg. Technischen Hochschule, Physikstrasse 5, CH-8044 Zürich.

Separatdruck aus der Schweiz. Zeitschrift für Hydrologie 31, Fasc. 1 (1969) Herausgegeben mit Unterstützung der Stiftung der Wirtschaft zur Förderung des Gewässerschutzes in der Schweiz 